\title{
A Levinson theorem for scattering from a Bose-Einstein condensate
}

\author{
J. Brand, I. Häring, and J.-M. Rost \\ Max Planck Institute for the Physics of Complex Systems, \\ Nöthnitzer Straße 38, 01187 Dresden, Germany
}

(Dated: November 17, 2018)

\begin{abstract}
A relation between the number of bound collective excitations of an atomic Bose-Einstein condensate and the phase shift of elastically scattered atoms is derived. Within the Bogoliubov model of a weakly interacting Bose gas this relation is exact and generalises Levinson's theorem. Specific features of the Bogoliubov model such as complex-energy and continuum bound states are discussed and a numerical example is given.
\end{abstract}

PACS numbers: $03.75 . \mathrm{Hh}, 03.65 . \mathrm{Nk}, 34.50-\mathrm{s}$

Exact results in physics are rare. One such result is Levinson's theorem [1], relating the number of bound states of a given potential to the accumulated scattering phase shift at threshold. This theorem was first formulated for the single-particle Schrödinger equation and in general does not hold for many-body systems. Instead, a generalisation for multi-channel scattering exists 2], relating the cumulative sum of the phase shifts of all open channels to the number of bound states. Little can be said about phase shifts in individual channels.

In this Letter, we consider the scattering of identical atoms from a spherically symmetric, weakly-interacting atomic Bose-Einstein condensate (BEC), held in a finite, localised trapping potential. In this situation, the phase shift $\delta^{l}(k)$ of $l$-wave scattering at momentum $k=0$ can be related to the number $n_{c}$ of bound collective excitations of the condensate by

$$
\delta^{l}(0)=\pi\left(n_{c}+\delta_{l 0}+\sigma / 2\right),
$$

where $\sigma=1$ for a bound state exactly at the threshold for $s$-wave scattering and $\sigma=0$ otherwise. Equation (1) is the generalisation of Levinson's theorem to the Bogoliubov equations describing the excitations of a weakly interacting BEC and is the main result of this Letter.

Scattering of cold atoms is a fundamental physical process relevant to high-precision atomic spectroscopy and quantum information processing. As temperatures are lowered, condensation of bosonic atoms is inevitable and the scattering of single atoms from condensates needs to be understood. Scattering experiments involving condensates have already been demonstrated in the context of four-wave mixing experiments 3, 4]. With the development of atomic lasers [5] as coherent matter wave sources and with the flexibility introduced by trapping and guiding of cold atoms with micro-fabricated electrical circuits [6, 7], precision measurement of scattering properties becomes feasible. Moreover, interferometric measurements should allow a direct access of the phase shifts and thereby confirm Levinson's theorem experimentally in contrast to the case of conventional atomic scattering experiments where usually intensities are measured only.
Theoretical attention has been given to identical particle scattering from BECs at low energy where transparency effects have been predicted [8] and at high energy where density distributions [9] and quantum correlations can be probed [10]. Very recently, negative time delays in one-dimensional scattering from atomic BECs have been predicted 11].

In the following, we will apply a multi-channel scattering formalism to the Bogoliubov equations and prove the relation (1) by contour integration. We will discuss special situations that can occur like unstable complexenergy collective modes and continuum bound states. An instructive numerical example of a realistic scattering situation is given and the role of the condensate and channel coupling for the Levinson theorem are discussed.

In the standard Bogoliubov approach [12], the weakly interacting Bose gas is described by a condensate with a small amount of coherent quantum depletion and a gas of non-interacting quasi-particles describing small thermal or externally induced collective excitations. The quasiparticles are mixtures of particle and hole excitations as long as they are located in the condensate region. Outside they just become free particles and can be identified with elastically scattered identical atoms. The Bogoliubov picture relies on the number $N$ of condensate atoms being large; indeed, the rate of inelastic scattering on a BEC decays exponentially in the Born approximation as $N$ grows large [9].

The quasi-particle energies $\epsilon_{\nu l}$ and the particle $(u)$ and hole $(v)$ amplitudes are obtained by solving the Bogoliubov equations for each partial wave $l[8]$ :

$$
\begin{aligned}
& \left(T_{l}+V_{11}\right) u_{\nu l}(r)-V_{12} v_{\nu l}(r)=\left(\epsilon_{\nu l}+\mu\right) u_{\nu l}(r) \\
& \left(T_{l}+V_{22}\right) v_{\nu l}(r)-V_{21} u_{\nu l}(r)=\left(-\epsilon_{\nu l}+\mu\right) v_{\nu l}(r),
\end{aligned}
$$

where $T_{l}=-\hbar^{2} /(2 m) \partial_{r r}+l(l+1) /\left(2 m r^{2}\right)$ is the kinetic energy with the atomic mass $m$. The potential is the same in both channels $V_{11}=V_{22}=V_{\text {trap }}(r)+2 g n(r)$ and so is the off-diagonal coupling $V_{12}=V_{21}=g n(r)$. The condensate density $n(r)$ and the chemical potential $\mu$ are determined by solutions of the stationary radial 
Gross-Pitaevskii equation:

$$
\left\{-\frac{\hbar^{2}}{2 m} \partial_{r r}+V_{\text {trap }}(r)+g N \frac{|\varphi(r)|^{2}}{4 \pi r^{2}}\right\} \varphi(r)=\mu \varphi(r),
$$

where $g \equiv 4 \pi \hbar^{2} a_{s} / m, a_{s}$ is the $s$-wave scattering length, and the condensate order parameter $\varphi(r)$ is assumed real and normalised to one. Here we make use of the scattering length approximation for the interatomic interaction and assume zero temperature, where the density $n(r)=N \varphi(r)^{2} /\left(4 \pi r^{2}\right)$. Both these assumptions are taken for simplicity. Generalisation to finite temperature Hartree-Fock-Bogoliubov schemes and a more elaborate treatment of interactions are possible and straightforward as long as the structure of Eqs. (2) is preserved. The trapping potential $V_{\text {trap }}(r)$ is a finite localised well that falls off to zero sufficiently quickly for large $r$ [19] and traps the condensate, leading to a negative chemical potential $\mu<0$. Formally, the Bogoliubov equations (2) can be understood as scattering equations for two coupled channels $u$ and $v$. At $\epsilon_{\nu l}+\mu>0$ there is a scattering continuum where the $u$ channel is open and the $v$ channel is closed. At large distance $r$, therefore, the hole amplitude $v_{\nu l}(r)$ decays exponentially while the asymptotic form

$$
u_{\nu l} \rightarrow \sin \left[k r+l \pi / 2+\delta^{l}(k)\right] \quad \text { as } \quad r \rightarrow \infty
$$

of the particle amplitude defines the phase shift $\delta^{l}(k)$ of the scattered atom with wavenumber $k=$ $\sqrt{2 m / \hbar^{2}\left(\epsilon_{\nu l}+\mu\right)}$. Note that there is a second scattering continuum for $-\epsilon_{\nu l}+\mu>0$ where the $v$ channel is open. It is related to the first continuum by the general symmetry of the Bogoliubov equations (2) that allows one to construct new solutions by interchanging $u$ and $v$ and simultaneously changing the sign of $\epsilon$. This property can be traced to the invariance of the coupling matrix $V$ under exchange of diagonally opposite matrix elements

$$
V_{12} \leftrightarrow V_{21} \quad \text { and } \quad V_{11} \leftrightarrow V_{22}
$$

We now proceed with the proof of Eq. (1) for the special case $l=0$. To this end, we slightly generalise the Bogoliubov equations (2) by introducing the channel momenta $k_{1}$ and $k_{2}$ as independent variables and write in matrix notation

$$
\left(-\partial_{r r} \mathbb{1}+V\right) \phi=K^{2} \phi
$$

where $K=\operatorname{diag}\left(k_{1}, k_{2}\right)$ and $\phi=\left[u_{\nu}(r), v_{\nu}(r)\right]^{T}$. We have now chosen units where $\hbar^{2} /(2 m)=1$ and dropped the channel index $l$. Analytical properties of multi-channel scattering solutions are most conveniently discussed by analysing the Fredholm determinant $\Delta\left(k_{1}, k_{2}\right)$, which generalises the Jost function of single-channel scattering [2]. We define the Fredholm determinant by $\Delta\left(k_{1}, k_{2}\right)=$ $\operatorname{det} \mathcal{F}$ with the Jost matrix $\mathcal{F}$

$$
\mathcal{F}=\mathbb{1}+\int_{0}^{\infty} e^{i K r} V(r) \Phi(K, r) d r .
$$

Here, the columns of the matrix $\Phi(K, r)$ are regular solutions of Eq. (6). $\Phi(K, r)$ obeys the system of coupled integral equations

$$
K \Phi(K, r)=\sin (K r)+\int_{0}^{r} \sin \left[K\left(r-r^{\prime}\right)\right] V\left(r^{\prime}\right) \Phi\left(K, r^{\prime}\right) d r^{\prime} .
$$

The Fredholm determinant $\Delta\left(k_{1}, k_{2}\right)$ is an entire function of $k_{1}$ and $k_{2}$ for finite range couplings. Under weaker conditions [19] the function $\Delta\left(k_{1}, k_{2}\right)$ is still analytic for $\Im k_{1}>0$ and $\Im k_{2}>0$.

The function $\Delta\left(k_{1}, k_{2}\right)$ contains the complete information about the physical scattering process and allows one to construct the $\mathbf{S}$ matrix. In particular, if $k_{1}$ or $k_{2}$ are real

$$
\Delta\left(k_{1}, k_{2}\right)=|\Delta| e^{-i \delta}
$$

is directly related to the phase shift $\delta$ of the open channel. Furthermore, we have the symmetry

$$
\Delta\left(k_{1}, k_{2}\right)=\Delta^{*}\left(-k_{1}^{*},-k_{2}^{*}\right),
$$

which follows for a real symmetric coupling matrix $V_{i j}$, and for $\Im k_{1} \geq 0$ and $\Im k_{2} \geq 0$, the relations

$$
\Delta\left(k_{1}, k_{2}\right) \rightarrow 1 \quad \text { as } \quad\left|k_{1}\right|,\left|k_{2}\right| \rightarrow \infty
$$

hold 2]. The symmetry

$$
\Delta\left(k_{1}, k_{2}\right)=\Delta\left(k_{2}, k_{1}\right)
$$

is a special property of the Bogoliubov equations and will be instrumental for the proof of Eq. (11). Equation (12) can be derived from the symmetry (5) using Eqs. (7) and (8).

We now return to the Bogoliubov equations (2) where the channel momenta $k_{1}$ and $k_{2}$ are not independent variables but related through $k_{1}^{2}+k_{2}^{2}=2 \mu$. Resolving for $k_{1}$, we specifically choose

$$
k_{2}\left(k_{1}\right)=i \sqrt{k_{1}^{2}-2 \mu}
$$

where $\sqrt{\cdot}$ denotes the usual positive square root with a branch cut along the negative real axis. If $k_{2}$ is given by (13) we say that $k_{1}$ is in the physical sheet. The choice (13) assures that $\Im k_{2}>0$, whenever $\Im k_{1}>0$. We see that $\Delta\left(k_{1}\right) \equiv \Delta\left(k_{1}, k_{2}\left(k_{1}\right)\right)$ is analytic in the upper complex half plane, except for a branch point at $k_{1}=$ $i \sqrt{-2 \mu}$ and a cut along the imaginary axis where $k_{1} / i \geq$ $\sqrt{-2 \mu}$ originating from the square root (13) as shown in Fig. 11 This is exactly where $k_{2}^{2}$ becomes positive, i.e. the $v$ channel is open and supports scattering solutions.

Bound solutions of the Bogoliubov equations correspond to zeros of $\Delta\left(k_{1}\right)$ in the upper half of the physical sheet where $\Im k_{1} \geq 0$. Solutions with positive quasiparticle energies $\epsilon_{\nu}$, the usual case for stable ground 


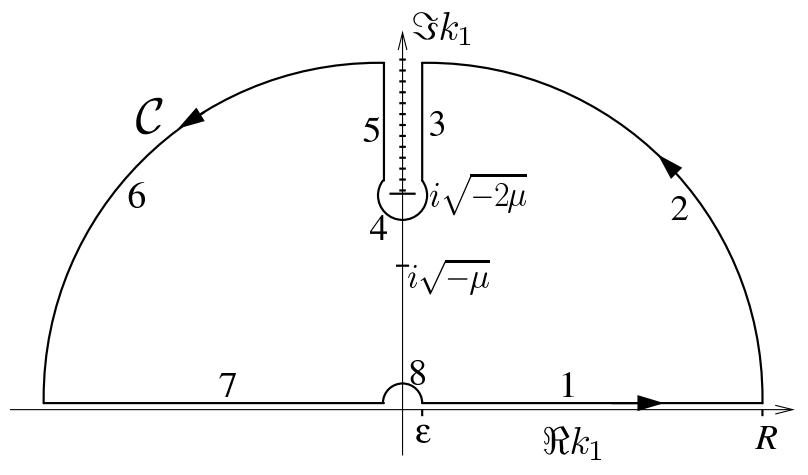

FIG. 1: Analytic structure of $\Delta\left(k_{1}\right)$ in the upper half of the physical sheet and integration contour $\mathcal{C}=\sum_{i=1}^{8} \mathcal{C}_{i}$ as detailed in the text. The segments $\mathcal{C}_{7}, \mathcal{C}_{1}$ and $\mathcal{C}_{3}, \mathcal{C}_{5}$ lie exactly on the real and imaginary axis, respectively.

state condensates, are found on the imaginary axis at $k_{1} / i \in[0, \sqrt{-\mu}]$. Due to the symmetry (5) of the Bogoliubov equations, every bound state at $k_{1} / i=\sqrt{-\epsilon_{\nu}-\mu}<$ $\sqrt{-\mu}$ has an image at $k_{1} / i=\sqrt{+\epsilon_{\nu}-\mu}>\sqrt{-\mu}$. Physically, only the solution with positive quasiparticle energy is meaningful. At $k_{1}=i \sqrt{-\mu}$ corresponding to zero quasiparticle energy $\epsilon_{0}=0$, a trivial bound solution of the Bogoliubov equations causes a double zero of $\Delta\left(k_{1}\right)$. This solution with $u_{0}=v_{0} \propto \varphi(r)$ is proportional to the condensate order parameter and does not describe a condensate excitation. In fact, quasiparticle excitations are confined to the orthogonal complement of $\varphi(r)$.

following integral in the complex $k_{1}$-plane over the

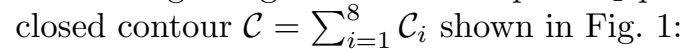

$$
\int_{\mathcal{C}} d \ln \Delta\left(k_{1}\right)=2 \pi i n_{z} \equiv 2 \pi i\left(2 n_{c}+2\right),
$$

which counts the number $n_{z}$ of zeros of $\Delta\left(k_{1}\right)$ with their multiplicity within the region enclosed by $\mathcal{C}$. From the preceeding discussion it is clear, that $n_{z}$ is given by twice the number of bound collective excitations $n_{c}$ enclosed by $\mathcal{C}$ plus the contribution from the trivial solution. Note that the contour $\mathcal{C}$ is more complicated than in the proof of the ordinary Levinson theorem, due to the cut and the branch point which has to be avoided.

We will now discuss contributions to the integral on the left hand side of Eq. (14). As in the usual proof of the Levinson theorem, the contributions on the negative and positive imaginary axis can be related to the scattering phase shift $\delta$ and with Eqs. (91) and (10) we obtain

$$
\int_{\mathcal{C}_{7}+\mathcal{C}_{1}} d \ln \Delta\left(k_{1}\right)=2 i[\delta(\varepsilon)-\delta(R)] .
$$

The small semicircle $\mathcal{C}_{8}$ of radius $\varepsilon$ around the origin gives a vanishing contribution for small $\varepsilon$ as do the quarter circles $\mathcal{C}_{2}$ and $\mathcal{C}_{6}$ for large $R$ due to Eq. (11).

The remaining contributions of the integrals over $\mathcal{C}_{3}$, $\mathcal{C}_{4}$, and $\mathcal{C}_{5}$ along the imaginary axis can be related to the contributions along the real axis due to the special structure of the Bogoliubov equations. In fact, Eq. (13) defines a conformal mapping, which maps the segments $\mathcal{C}_{3}, \mathcal{C}_{4}$, and $\mathcal{C}_{5}$ of the $k_{1}$ plane onto the segments $\mathcal{C}_{7}, \mathcal{C}_{8}$, and $\mathcal{C}_{1}$ of the $k_{2}$ plane, respectively. With the symmetry (12) we obtain $\int_{\mathcal{C}_{3}} d \ln \Delta\left(k_{1}\right)=\int_{\mathcal{C}_{7}} d \ln \Delta\left(k_{1}\right)$ and likewise $\int_{\mathcal{C}_{4}}=\int_{\mathcal{C}_{8}}$ and $\int_{\mathcal{C}_{5}}=\int_{\mathcal{C}_{1}}$.

We have now evaluated the left hand side of Eq. (14), which becomes $4 i[\delta(0)-\delta(\infty)]$ in the limits $\varepsilon \rightarrow 0^{+}$and $R \rightarrow \infty$. Note that we can set $\delta(\infty)=0$ due to Eq. (11). Thus we obtain Eq. (1) for $l=0$ and under the assumption that there are no zeros of $\Delta\left(k_{1}\right)$ on the real axis. The generalisation of this proof for $l>0$ is straightforward within the partial-wave formalism. However, for $l>0$ all zeros enclosed by the contour $\mathcal{C}$ correspond to collective excitations since the modes $u$ and $v$ are always orthogonal to the condensate wavefunction $\varphi$.

Finally, we consider the possibility of zeros of $\Delta\left(k_{1}\right)$ on the real axis. Additionally to zeros at the threshold $k_{1}=0$, also encountered in single-channel scattering, we cannot exclude the possibility of zeros of $\Delta\left(k_{1}\right)$ at real $k_{1} \neq 0$. If zeros of $\Delta\left(k_{1}\right)$ on the real axes exist, they fully contribute to a continuously measured phase shift in the same way as continuum bound states do in conventional multi-channel scattering theory with the exception of a zero at $k_{1}=0$ in the $l=0$ channel [2]. This specific case is known as a half bound state as it only contributes $\pi / 2$ to the phase shift, hence the term $\sigma / 2$ in Eq. (11).

So far we have assumed that the condensate is in a stable ground state of the trap, in which case all bound excitations described by the Bogoliubov equations have finite pseudo norm $\eta \equiv \int\left(|u|^{2}-|v|^{2}\right) d r \neq 0$ and those with $\eta>0$ have positive quasi-particle energy $\varepsilon_{\nu}$. However, this restriction is not necessary. For excited states $\varphi(r)$ of the stationary Gross-Pitaevskii equation (3), anomalous solutions of the Bogoliubov equation are possible with $\epsilon_{\nu} \leq 0$ and $\eta>0$. Such anomalous modes are known to occur for solitons in highly elongated traps [13, 14] and for vortices [15]. Furthermore, bound solutions with complex $\epsilon_{\nu}$ and $\eta=0$ may occur, which correspond to zeros of $\Delta\left(k_{1}\right)$ on the physical sheet but off the imaginary axis. These solutions arise when the condensate is in a stationary but unstable excited state of the GrossPitaevskii equation and describe modes of exponential decay, predicted and seen, e.g., for dark solitons [16, 17] and attractive condensates [18]. These unstable complex modes are not to be confused with scattering resonances, which have zeros below the real axis and in the unphysical sheet. Both anomalous and complex Bogoliubov modes contribute to the contour integral (14) and thus are predicted to be visible in the build up of the elastic scattering phase shift $\delta(0)$, which can, in principle, be probed experimentally.

This is exemplified in Fig. 2 for a ground-state BEC of 2000 Rubidium atoms. Note that the relevant range of 


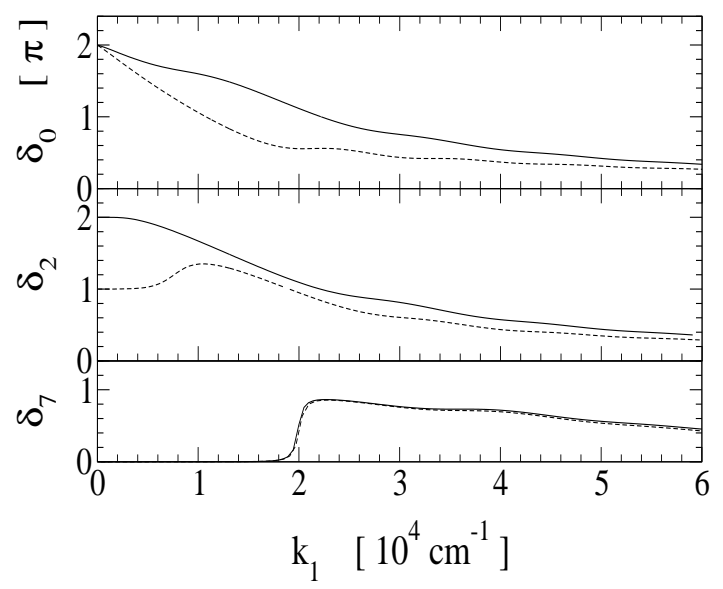

FIG. 2: Phase shifts $\delta_{l}$ from the full solution of Eqs. (2) (solid), simple potential scattering without coupling $\left[V_{12}=0\right.$ in Eqs. (2)] (dashed). In the example, 2000 Rubidium atoms are held in a finite harmonic trap of frequency $\omega=2 \pi 200 \mathrm{~s}^{-1}$ and depth $V_{0}=8.0 \hbar \omega$ resulting in the chemical potential $\mu=-3.34 \hbar \omega$ and allowing for up to two bound collective excitations for $1 \leq l \leq 5$.

scattering energies corresponding to temperatures of the order of $10 \mathrm{nK}$ is within the accessible range of current cold-atom experiments. For $l=0$ one bound collective excitation and the trivial solution account for a phase shift at threshold $\delta_{0}(0)$ of $2 \pi$ both in the full Bogoliubov equations (solid) and in the potential scattering approximation (dashed) where we have set $V_{12}=0$. Both solutions coincide in the absence of a bound mode for $\delta_{7}\left(k_{1}\right)$ which exhibits, instead, a resonance caused by a zero of $\Delta\left(k_{1}\right)$ with $k_{1}$ having a small negative imaginary part. The interesting case is $\delta_{2}\left(k_{1}\right)$ which indicates one more bound state in the full solution compared to simple potential scattering.

The offdiagonal coupling $V_{12}$ is of attractive nature and binds extra states although the interparticle interaction is repulsive in both examples. For large scattering momenta and large angular momenta this coupling to the $v$ mode becomes less important, as the details of the interaction region are hardly probed. Figure 3 shows the number of bound states of a square well model system. As the well is filled up with repulsive atoms, fewer collective excitations are bound. Note that for $\Gamma>V_{0} / 6$ the trapped BEC has bound quasiparticle states only due to the coupling between hole and particle modes.

We have presented a Levinson theorem relating the number of bound collectively excited states of an interacting many-body system, a BEC, to the phase shifts of single-particle scattering. With the possibilities of cold atom scattering and interference, we can expect to see a direct experimental verification of a fundamental theorem of mathematical physics. However, our derivation is based on a weakly interacting Bose gas without inelastic scattering processes. It should be seen as a challenge both to experiments and theory to find the corrections to Eq. (1) in a real interacting system. Dilute-gas atomic BECs are an ideal system for this because the experimental set up is stupendously manageable.

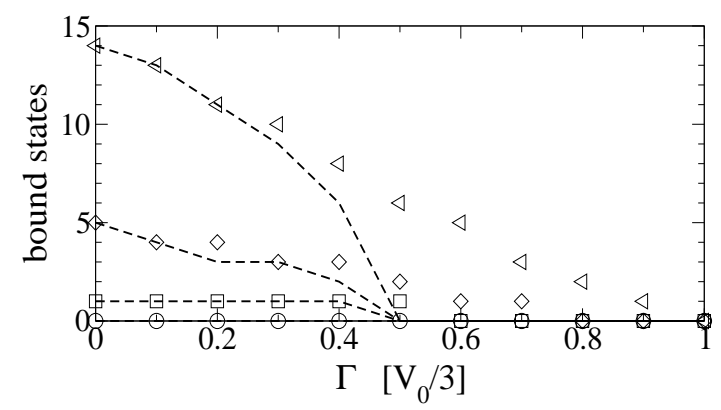

FIG. 3: Number of bound collective modes for a square-well of depth $V_{0}=10,100,1000 \hbar^{2} / m a^{2}$, respectively, and radius $a$ with a Thomas-Fermi approximation for the wave function as a function of the nonlinear coupling $\Gamma=N a_{s} / a$. Full solution (symbols), potential scattering (dashed) as in Fig. 2

[1] N. Levinson, K. Dan. Vidensk. Selsk. Mat. Fys. Medd. 25, 1 (1949).

[2] R. G. Newton, Scattering Theory of Waves and Particles, 2nd ed. (Springer-Verlag, New York, 1982).

[3] L. Deng et al., Phys. Rev. Lett. 83, 5407 (1999).

[4] J. M. Vogels, K. Xu, and W. Ketterle, Phys. Rev. Lett. 89, 020401 (2002).

[5] I. Bloch, T. W. Hänsch, and T. Esslinger, Phys. Rev. Lett. 82, 3008 (1999).

[6] D. Cassettari et al., Phys. Rev. Lett. 85, 5483 (2000).

[7] H. Ott et al., Phys. Rev. Lett. 87, 230401 (2001).

[8] A. Wynveen et al., Phys. Rev. A 62, 023602 (2000).

[9] Z. Idziaszek, K. Rzazewski, and M. Wilkens, J. Phys. B 32, L205 (1999).

[10] A. B. Kuklov and B. V. Svistunov, Phys. Rev. A 60, R769 (1999).

[11] U. V. Poulsen and K. Mølmer, Phys. Rev. A 67, 013610 (2003).

[12] F. Dalfovo, S. Giorgini, L. P. Pitaevskii, and S. Stringari, Rev. Mod. Phys. 71, 463 (1999).

[13] A. Muryshev, H. van Linden van den Heuvell, and G. Shlyapnikov, Phys. Rev. A 60, R2665 (1999).

[14] J. Dziarmaga and K. Sacha, Phys. Rev. A 66, 043620 (2002).

[15] A. L. Fetter and A. A. Svidzinsky, Journal of Physics: Condensed Matter 13, R135 (2001).

[16] B. Anderson et al., Phys. Rev. Lett. 86, 2926 (2001).

[17] J. Brand and W. P. Reinhardt, Phys. Rev. A 65, 043612 (2002).

[18] K. E. Strecker et al., Nature 417, 150 (2002).

[19] We require $\int_{0}^{\infty}\left|V_{\text {trap }}(r)\right| r^{n} d r<\infty$ with $n=1,2$ as usual in scattering theory. 